\title{
Protective and Indicating Effect of Indirect Bilirubin in Intracranial or Extracranial Artery Atherosclerotic Stenosis Progresses
}

\author{
Yingzhu Chen ${ }^{1 *}$, Xianxian Zhang2*, Lingling Zhang1*\#, Rongyin Qin ${ }^{1}$, Kangping Song1 ${ }^{1}$ Lu Xiao ${ }^{1}$ \\ ${ }^{1}$ Department of Neurology, Clinical Medical College, Yangzhou University, Yangzhou, China \\ ${ }^{2}$ Department of Neurology, Yancheng Third People's Hospital, Yancheng, China \\ Email: "LLxiangwang@163.com
}

Received 30 June 2015; accepted 20 July 2015; published 23 July 2015

Copyright (C) 2015 by authors and Scientific Research Publishing Inc.

This work is licensed under the Creative Commons Attribution International License (CC BY).

http://creativecommons.org/licenses/by/4.0/

(c) (i) Open Access

\section{Abstract}

Background: Bilirubin is the metabolic end-product of heme degradation by heme oxygenase (HO), which has recently been shown to act as an antioxidant which can protect against atherosclerosis. This study explored the relationship between serum bilirubin levels and different degrees of atherosclerotic stenosis in intracranial or extracranial arteries. Methods: The study included 189 patients undergoing digital subtraction angiography (DSA) diagnosed as being normal or having been confirmed as atherosc lerotic stenosis in the intracranial or extracranial arteries. The patients were allocated to normal, mild ( $<50 \%$ diameter stenosis), moderate (50\% - 69\% stenosis), severe $(70 \%$ - 99\% stenosis) and occlusion groups according to the severity of stenosis proved by DSA. Blood samples were collected to determine bilirubin concentrations and other biochemical indicators of atherosclerosis. Univariate and multivariate analyses were performed to evaluate the associations between disease severity and biomarkers. Results: Indirect bilirubin (Ibil) concentrations increased in parallel with the increasing severity of atherosclerotic stenosis in the intracranial or extracranial arteries, but decreased in patients with occluded cranial vessels. Multivariate analysis showed that Ibil levels were significantly higher in patients with severe stenosis group than in those with mild stenosis (0R, 1.464; 95\% CI, $1.050-2.042 ; P=0.024$ ). However, Ibil levels were significantly lower in patients with occlusion than in those with severe stenosis (OR, 0.790; 95\% CI, $0.684-0.913 ; P=$ 0.001). Conclusions: Ibil appears to have a protective effect against the development of atherosclerotic stenosis in intracranial or extracranial arteries. The biosynthesis of Ibil increases with stenosis progresses but decreases once occlusion occurs.

\footnotetext{
*These authors contribute equally to the paper.

"Corresponding author.
}

How to cite this paper: Chen, Y.Z., Zhang, X.X., Zhang, L.L., Qin, R.Y., Song, K.P. and Xiao, L. (2015) Protective and Indicating Effect of Indirect Bilirubin in Intracranial or Extracranial Artery Atherosclerotic Stenosis Progresses. International Journal of Clinical Medicine, 6, 512-519. http://dx.doi.org/10.4236/ijcm.2015.67069 


\section{Keywords}

\section{Indirect Bilirubin, Atherosclerotic Stenosis, Intracranial Arteries, Extracranial Arteries}

\section{Introduction}

Intracranial atherosclerotic stenosis is one of the most common causes of ischaemic cerebrolvascular disease worldwide. Atherosclerotic stenosis of the internal carotid artery can lead to $10 \%-15 \%$ of all strokes [1]. $70 \%$ of symptomatic intracranial atherosclerotic stenosis is responsible for a high risk of recurrent stroke [2]. In 2005, the incidence of stroke among patients from Germany who had symptomatic stenosis was reported at $7.3 \%$ per year [3]. It has estimated a $26 \%$ increase in the risk of ischemic cerebrovascular disease for every $10 \%$ increase in the degree of stenosis [4]. Intracranial atherosclerotic stenosis (ICAS) is associated with $8 \%$ to $10 \%$ of all ischemic strokes in the United States, but accounts for 33\% to 54\% of all ischemic strokes in Asia. In China, ICAS may be the cause of $37 \%$ to $51 \%$ of all strokes or transient ischemic attacks (TIA) [5]. These statistics highlight the importance of understanding the mechanisms involved in the development and elimination of atherosclerotic stenosis.

Bilirubin is generated by the action of the enzyme biliverdin reductase on biliverdin in the catabolism of heme [6], which contains indirect bilirubin (Ibil) and direct bilirubin (Dbil). Ibil is a bile pigment produced during the degredation of hemoproteins. Ibil is a hydrophobic compound with extremely low water solubility and high lipid solubility, which is transported bound to serum albumin to the liver conjugated with glucuronic acid, converted to Dbil in the hepatocytes by the enzyme UGT1A1 and finally secreted into the bile to be eliminated [7].

It was once believed that bilirubin was only the metabolic end-product of heme degradation by HO, but has now emerged as an important endogenous anti-inflammatory and antioxidant molecule [8]. It is increasingly appreciated that bilirubin has strong anti-oxidative properties, which owe its ability to scavenge peroxyl radicals and to inhibit low density lipoprotein (LDL) oxidation [9]. Recent data have demonstrated that mildly elevated serum bilirubin is associated with a reduced prevalence of oxidative stress-mediated disease [10]. Serum bilirubin may prevent experimental atherosclerosis possibly by the scavenging of oxygen radicals and by its inhibitory effects against LDL oxidation [9].

Some cross-sectional and prospective studies have reported negative associations between bilirubin levels and coronary artery disease (CAD) [11], peripheral vascular disease, carotid intimal-medial thickness, stroke, nonalcoholic fatty liver and metabolic syndrome [12]. The first indication that serum bilirubin levels might be related to atherosclerosis was reported in 1994 [13], by a study that demonstrated an inverse relationship between serum bilirubin and CAD. A later study, which included 1741 Japanese subjects, reported that each $1.7 \mu \mathrm{mol} / \mathrm{L}$ $(0.1 \mathrm{mg} / \mathrm{dL})$ increased in bilirubin decreased the risk of carotid artery plaque formation by $3.7 \%$ [14]. In another study every $1.7 \mu \mathrm{mol} / \mathrm{L}(0.1 \mathrm{mg} / \mathrm{dL})$ increase in serum bilirubin was associated with a $6 \%$ reduction in the odds of developing peripheral artery disease [15]. Other workers reported that men in the highest quartile of serum bilirubin concentrations had a lower hazard ratio for ischemic stroke than men in the lowest quartile of bilirubin concentrations $(P=0.016)$ [16]. Patients with high levels of total bilirubin (Tbil) have also been shown to exhibit a lower rate of coronary stent restenosis than patients with low Tbil [17].

Few studies have explored the possible associations between serum bilirubin and atherosclerotic stenosis in the intracranial or ertracranial arteries. In this study we analyzed serum bilirubin levels in patients with different degrees of angiographically proved atherosclerotic stenosis in intracranial or extracranial arteries.

\section{Methods}

\subsection{Study Population and Design}

We consecutively recruited 189 patients undergoing digital subtraction angiography (DSA) at the Department of Neurology in the Medical College of Yangzhou University, China between 1 April 2009 and 31 March 2012. All participants gave written informed consent. The study was approved by the research ethics committee of Clinical Medical College of Yangzhou University. The approved number was 2009023. The analysis included patients with atherosclerotic stenosis proved by DSA in intracranial or extracranial arteries, as well as those 
whose DSA examination was normal. We excluded patients with intracranial or subarachnoid hemorrhage as well as those with aneurism, Moyamoya disease, artery dissection, arteriovenous malformation, arteriovenous fistula; or an established history of bilirubin metabolism abnormity.

All patients were allocated to five groups according to degrees of atherosclerotic stenosis in intracranial or extracranial arteries. Severity of stenosis was based on the European Carotid Surgery Trial (ECST) criteria [18] as follows: normal, mild ( $<50 \%$ diameter stenosis), moderate ( $50 \%$ - $69 \%$ stenosis), severe ( $70 \%$ - $99 \%$ stenosis) and occlusion. The highest percentage of cerebral artery stenosis was used for each evaluation.

The following risk factors were evaluated in all patients: age, gender, hypertension, diabetes mellitus, cigarette smoking, alcohol consumption and other biochemical markers which were potential predictors according to previous reports.

Hypertension was defined as a preadmission history of hypertension, with or without treatment, or by an average systolic blood pressure (SBP) $\geq 140 \mathrm{mmHg}$ or diastolic blood pressure (DBP) $\geq 90 \mathrm{mmHg}$, that was sustained for $\geq 2$ weeks after hospital admission and confirmed during follow-up. Diabetes mellitus was defined as a preadmission history of diabetes or as fasting venous plasma glucose values $\geq 7.0 \mathrm{mmol} / \mathrm{L}$ on at least two separate occasions, and/or as a blood glucose levels $\geq 11.1 \mathrm{mmol} / \mathrm{L}$ on two occasions $2 \mathrm{~h}$ after intake of $75 \mathrm{~g}$ of oral glucose [8]. Cigarette smoking was defined as daily use of $\geq 10$ cigarettes during the previous 6 months; and alcohol consumption defined as the consumption of $\geq 2$ alcoholic drinks per day.

\subsection{Digital Subtraction Angiography and Image Analysis}

DSA was performed using a GE Innova Angiography machine (Innova 3100 IQ; Advantage 4.4; GE Medical Systems, America). A transfemoral approach using the Seldinger technique with a 5F catheter was adopted by all patients. Nonionic iodinate contrast medium was administered using a Liebel-Flarsheim pressure injector. Images at the level of aortic were obtained by administration of $20 \mathrm{~mL}$ of contrast agent at an injection rate of 10 to $15 \mathrm{~mL} / \mathrm{s}$. For selective catheterization at the level of the common carotid arteries an injection volume of 7 to 8 $\mathrm{mL}$ was administered at a rate of 5 to $6 \mathrm{~mL} / \mathrm{s}$ and for visualization of the vertebral artery 5 to $6 \mathrm{~mL}$ of contrast agent was administered at a rate of 2 to $3 \mathrm{~mL} / \mathrm{s}$.

The first injection of contrast agent was made at the level of the aortic arch and subsequent left and right anterior oblique images were obtained identifying the common carotid arteries, internal carotid arteries, intracranial arterial and the vertebral arteries. The analysis of DSA images was performed in consensus by two neuroradiologists.

The narrowest lumen diameter was measured and converted to percentage of stenosis using the formula: maximal stenosis $=[1-($ minimal lumen diameter $/$ nominal lumen diameter $)] \times 100 \%$.

\subsection{Measurement of Serum Bilirubin and Blood Chemistry Parameters}

Routine blood and biochemical tests were performed in all patients. The blood samples were obtained for all patients from the cubital vein after a 12-hour overnight fast. The blood was collected into EDTA-containing tubes, and all plasma samples were stored at $-80^{\circ} \mathrm{C}$ until analysis [19]. Serum bilirubin concentrations and other hematological and biochemical markers (lipids, clotting factors, uric acid, blood cell count and proteins), were determined on an automatic analyzer (Modular DDPP; Roche; German).

\subsection{Statistical Analysis}

All statistical analyses were conducted using SPSS version 16.0 software (SPSS Inc., Chicago, IL). Data was collected retrospectively. Admission categorical variables were coded as 0/1 (absent or present) and expressed as counts and percentages. Continuous variables were expressed as means and standard deviations $( \pm \mathrm{SD})$.

For univariate analyses, overall frequencies or mean $\pm \mathrm{SD}$ values were compared using chi squared statistics for dichotomous variables. Continuous variables were analyzed using Student's $t$ tests for normally distributed and Mann-Whitney $U$ tests for non-normally distributed data.

Multivariate logistic regression analyses were performed to determine if bilirubin concentration was independently associated with the progression of stenosis. Age, sex, hypertension, diabetes mellitus and other variables with a $P$ value $<0.05$ on univariate analysis were included in the model. Results were expressed as adjusted 
odds ratios (ORs) with corresponding 95\% CI. All probability values were 2-sided, and $P<0.05$ were considered statistically significant.

\section{Results}

Among the 189 patients included in the study, 68 patients were diagnosed as TIA, 106 patients were diagnosed with acute ischemic stroke, 14 patients were chronic cerebral ischemia and 1 patient were sudden deafness. The population included 143 male and 46 female patients with a mean age of $63.1 \pm 9.8$ years (range: 36 to 81 years).

Patients were initially divided into a stenosis-group $(n=162)$ and non-stenosis group $(n=27$; Table 1$)$. Univariate analysis indicated that average age, cigarette smoking rate and platelets levels were significantly higher in the stenosis group than in the non-stenosis group $(P=0.007, P=0.025$ and $P=0.047$, respectively). Multivariate analysis (Table 2) indicated that the odds ratio associated with increasing age was 1.077 (95\% CI, 1.028 1.128), the Odds ratio associated with higher cigarette smoking rate was 1.127 (95\% CI, $1.102-1.202$ ) and the Odds ratio associated with a higher platelet count was 1.014 (95\% CI, 1.004 - 1.024). There were no significant differences between the stenosis and non-stenosis groups for any of the other variables including serum bilirubin levels. However both Tbil and Ibil appeared to be numerically lower in the non-stenosis group than in patients with stenosis.

Table 1. Potential baseline predictors of atherosclerotic stenosis.

\begin{tabular}{|c|c|c|}
\hline Predictors & Non-stenosis group $(\mathrm{n}=27)$ & Stenosis group $(\mathrm{n}=126)$ \\
\hline Age, years & $58.5 \pm 8.3$ & $63.9 \pm 9.8$ \\
\hline Sex, male, n (\%) & $18(66.7 \%)$ & 125 (77.1\%) \\
\hline Hypertension, n (\%) & $18(66.7 \%)$ & 125 (77.1\%) \\
\hline Diabetes mellitus, n (\%) & 7 (25.9\%) & $41(25.3 \%)$ \\
\hline Cigarette smoking, n (\%) & $11(40.7 \%)$ & $103(63.6 \%)$ \\
\hline Alcohol consumption, n (\%) & $9(33.3 \%)$ & 67 (41.4\%) \\
\hline Tbil, $\mu \mathrm{mol} / \mathrm{L}$ & $13.5 \pm 6.1$ & $13.1 \pm 6.3$ \\
\hline Dbil, $\mu \mathrm{mol} / \mathrm{L}$ & $3.4 \pm 1.5$ & $3.5 \pm 1.8$ \\
\hline Ibil, $\mu \mathrm{mol} / \mathrm{L}$ & $10.3 \pm 4.9$ & $9.8 \pm 4.9$ \\
\hline Uric acid, $\mu \mathrm{mol} / \mathrm{L}$ & $314.7 \pm 20.2$ & $328.8 \pm 96.9$ \\
\hline Triglycerides, mmol/L & $1.6 \pm 0.7$ & $1.7 \pm 0.8$ \\
\hline Total cholesterol, mmol/L & $4.5 \pm 0.9$ & $4.6 \pm 0.9$ \\
\hline HDL-C, mmol/L & $1.1 \pm 0.3$ & $1.1 \pm 0.3$ \\
\hline LDL-C, mmol/L & $2.7 \pm 0.7$ & $2.7 \pm 0.7$ \\
\hline Total bile acid, $\mu \mathrm{mol} / \mathrm{L}$ & $4.1 \pm 2.7$ & $4.5 \pm 4.1$ \\
\hline Pre albumin, mg/L & $210.9 \pm 53.7$ & $212.1 \pm 56.4$ \\
\hline WBC, $\times 10^{9} / \mathrm{L}$ & $6.7 \pm 2.1$ & $7.4 \pm 2.7$ \\
\hline Platelets, $\times 10^{9} / \mathrm{L}$ & $170.2 \pm 45.9$ & $192.5 \pm 54.6$ \\
\hline Prothrombin time, s & $13.4 \pm 0.8$ & $13.3 \pm 0.8$ \\
\hline APTT, s & $36.8 \pm 3.6$ & $39.0 \pm 6.0$ \\
\hline Fibrinogen, g/L & $3.0 \pm 0.5$ & $3.5 \pm 1.6$ \\
\hline
\end{tabular}

Values were presented as mean \pm SD or frequencies (\%). Tbil: total bilirubin levels; Dbil: direct bilirubin levels; Ibil: indirect bilirubin levels; APTT: activated partial thromboplastin time. 
Table 2. Predictors for atherosclerotic stenosis.

\begin{tabular}{cccc}
\hline Predictors & Odds ratio & 95\% CI & P-Value $^{*}$ \\
\hline Age,, $\mathrm{r}$ & 1.077 & $1.028-1.128$ & 0.002 \\
Sex, male & 2.235 & $0.874-5.715$ & 0.093 \\
Cigarette smoking, $\mathrm{n}$ & 1.127 & $1.102-1.202$ & 0.015 \\
Platelets, $\times 10^{9} / \mathrm{L}$ & 1.014 & $1.004-1.024$ & 0.007 \\
Tbil, $\mu \mathrm{mol} / \mathrm{L}$ & 1.072 & $0.670-1.715$ & 0.773 \\
Dbil, $\mu \mathrm{mol} / \mathrm{L}$ & 1.110 & $0.792-1.556$ & 0.546 \\
Ibil, $\mu \mathrm{mol} / \mathrm{L}$ & 0.956 & $0.852-1.073$ & 0.448 \\
\hline
\end{tabular}

The stenosis group was divided into four subgroups according to ECST criteria (Figure 1). Thirteen patients had mild stenosis, 27 had moderate stenosis, 55 patients had severe stenosis, and 67 had occlusion. Univariate analysis showed that the levels of Tbil and Ibil in the moderate and severe stenosis groups were significantly higher than those in the mild stenosis group. Multivariate analysis based on the four subgroups (Table 3) indicated that Ibil levels were significantly higher in the severe stenosis group than in the mild stenosis (OR, 1.464; 95\% CI, 1.050 - 2.042). However, Ibil levels in the occlusion group were significantly lower than those in the severe stenosis group (OR: 0.790; 95\% CI: 0.684 - 0.913).

\section{Discussion}

Bilirubin is a heme metabolite generated by action of heme oxygenase and biliverdin reductase enzymes. Bilirubin was believed to be a potentially toxic metabolite in the past decades, but more recent data have demonstrated a negative relationship between serum bilirubin levels and atherosclerosis [10] [20]. These studies have suggested that bilirubin has the potential to inhibit low-density lipoprotein oxidation and scavenge oxygen radicals [8]. It is also thought to have anti-inflammatory actions that affect the chemotaxis of monocytes. These processes may all play a crucial role in preventing the development of atherogenesis [21]-[24]. Bilirubin has also been shown to block the proliferation and migration of vascular smooth muscle cells [25] [26].

In the present study, we focused on the association between serum bilirubin levels and different degrees of atherosclerotic stenosis in intracranial or extracranial arteries. We found that serum bilirubin levels (including Tbil and Ibil) were lower in patients with stenosis group than in the non-stenosis group, but the differences were not statistically significant $(P>0.05)$. Previous researchers demonstrated that levels of serum bilirubin in patients with atherosclerosis were lower than those patients without it [13]-[17] [27]. The total patients enrolled in our study were diagnosed as ischemic cerebrovascular disease, and thus some patients in non-stenosis group might be accompanied by atherosclerosis in intracranial or extracranial artery, which might affect the result. For this reason the non-stenosis group was excluded from further multivariate analyses.

Among the four groups with stenosis, Ibil levels were significantly higher in patients with severe stenosis than in those with mild stenosis group, suggesting that the level of serum Ibil increased with the increasing severity of stenosis. Bilirubin had been proven to be a major contributor to the total antioxidant capacity in blood plasma [28] [29], and its biosynthesis had been known to be evoked by oxidative stress [30]. Thus, it was likely that the development of stenosis of cranial arteries caused the biosynthesis of Ibil to be increased. However, the Ibil levels in the occlusion group were significantly lower than those in the group with severe stenosis, suggesting that once the blood vessels had become occluded, the biosynthesis of Ibil would decrease. These findings suggested that with the progression of atherosclerotic stenosis, the human body would increase the levels of serum bilirubin to enhance the capacity of anti-oxidation, which could retard the process. Our research found that the main antioxidant activity was derived from Ibil. The levels of Dbil showed no significant differences among four groups. Dbil is synthesized by the combination of Ibil and glucuronic acid in the liver, suggesting that antioxidant capacity might be weakened by this conjugation reaction.

Our results indicated that monitoring Ibil concentrations in patients with established atherosclerotic stenosis of cranial arteries may be a way of predicting progression of stenosis if consecutive values showed a tendency to 


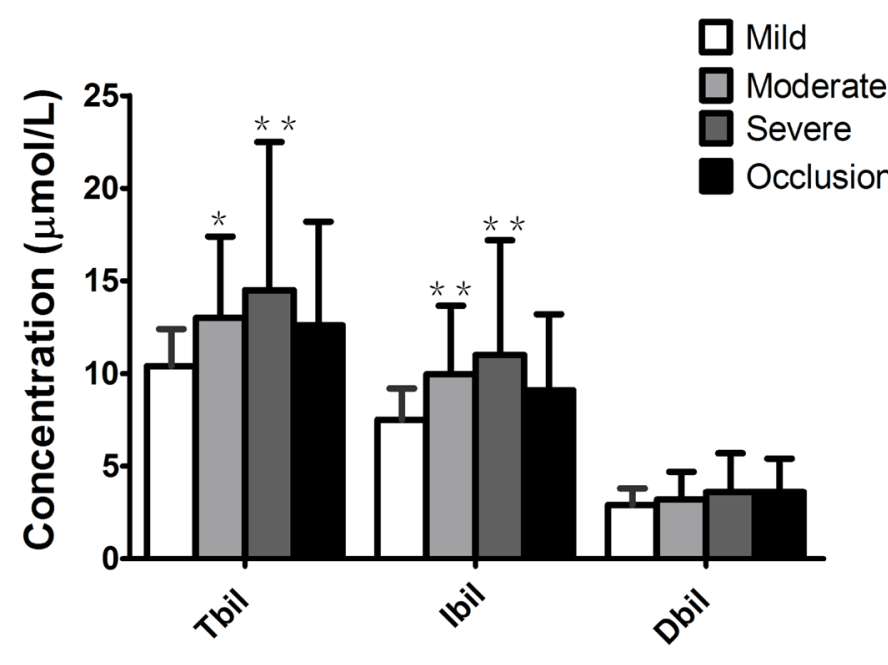

Figure 1. Bilirubin levels in patients with different degrees of stenosis. The stenosis group was subdivided into four subgroups according to ECST criteria. Thirteen patients had mild stenosis, 27 had moderate stenosis, 55 patients had severe stenosis, and 67 had occlusion. The serum concentrations of Tbil, Dbil, and Ibil were analysed by an automatic analyzer (Modular DDPP; Roche; German). The level of different bilirubin of each group comupted with Mean \pm SD. ${ }^{*} P<$ 0.05 moderate stenosis group versus mild stenosis group in Tbil, ${ }^{* *} P$ $<0.01$ severe stenosis group versus mild stenosis group in Tbil. ${ }^{* *} \mathrm{P}<$ 0.01 moderate and severe stenosis groups versus mild stenosis group in Ibil.

Table 3. The prognostic effect of Ibil levels on severity of stenosis.

\begin{tabular}{ccccc}
\hline Group & Ibil $(\mu \mathrm{mol} / \mathrm{L})$ & Odds ratio & $95 \%$ CI & $P_{\text {-Value }}^{*}$ \\
\hline Mild $(\mathrm{n}=13)$ & $7.5 \pm 1.7$ & & & $0.978-1.825$ \\
Moderate $(\mathrm{n}=27)^{\dagger}$ & $9.96 \pm 3.7$ & 1.332 & $1.050-2.042$ & 0.069 \\
Severe $(\mathrm{n}=55)^{\dagger}$ & $11.0 \pm 6.2$ & 1.464 & $0.684-0.913$ & 0.001 \\
Occlusion $(\mathrm{n}=67)^{\ddagger}$ & $9.1 \pm 4.1$ & 0.790 & & \\
\hline
\end{tabular}

increase; and we assumed that bilirubin may be a potent medication for prevention and treatment of atherosclerotic stenosis, as Robert Öllinger et al. reported [23].

Both univariate and multivariate analysis also identified age, platelet levels and cigarette smoking as independent risk factors for atherosclerotic stenosis. It suggested that these were important risk factors that participate in the formation of atherosclerosis stenosis and aggravate atherosclerosis stenosis, which were also consistent with previous reports [31] [32].

Our study was limited by its retrospective design and relatively small sample size. In addition, the trends identified in the study were based on different patients rather than on monitoring different stages of the disease in the same patients. The results of this study did, however, provide a basis for more rigorously designed prospective researchers with larger samples aimed at clarifying the possible inter-relationships between bilirubin levels and the progression of atherosclerotic stenosis. This novel finding must be confirmed in larger cohorts, and its clinical implications are worthy of further investigations.

\section{Acknowledgements}

The author thanks for Yingzhu Chen professor. They appreciate the help of the department of neurology and invasive technology, Clinical Medical College, Yangzhou University, Yangzhou, China. 


\section{References}

[1] Bonati, L.H., Dobson, J., Featherstone, R.L., Ederle, J., van der Worp, H.B., de Borst, G.J., Mali, W.P.T.M., Beard, J.D., Cleveland, T., Engelter, S.T., Lyrer, P.A., Ford, G.A., Dorman, P.J. and Brown, M.M. (2015) Long-Term Outcomes after Stenting Versus Endarterectomy for Treatment of Symptomatic Carotid Stenosis: The International Carotid Stenting Study (ICSS) Randomised Trial. The Lancet, 385, 529-538.

http://dx.doi.org/10.1016/S0140-6736(14)61184-3

[2] Ma, N., Xu, Z., Mo, D., Gao, F., Gao, K., Sun, X., Xu, X., Liu, L., Song, L., Wang, T., Zhao, X., Wang, Y., Wang, Y. and Miao, Z. (2014) Safety of Low-Dose Aspirin in Endovascular Treatment for Intracranial Atherosclerotic Stenosis. PloS One, 9, e105252. http://dx.doi.org/10.1371/journal.pone.0105252

[3] Kern, R., Steinke, W., Daffertshofer, M., Prager, R. and Hennerici, M. (2005) Stroke Recurrences in Patients with Symptomatic vs Asymptomatic Middle Cerebral Artery Disease. Neurology, 65, 859-864. http://dx.doi.org/10.1212/01.wnl.0000175983.76110.59

[4] Tegos, T.J., Kalodiki, E., Daskalopoulou, S.S. and Nicolaides, A.N. (2000) Stroke: Epidemiology, Clinical Picture, and Risk Factors-Part I of III. Angiology, 51, 793-808. http://dx.doi.org/10.1177/000331970005101001

[5] Qian, Y., Pu, Y., Liu, L., Wang, D.Z., Zhao, X., Wang, C., Wang, Y., Liu, G., Pan, Y. and Wang, Y. (2013) Low HDL-C Level Is Associated with the Development of Intracranial Artery Stenosis: Analysis from the Chinese IntraCranial Athero Sclerosis (CICAS) Study. PloS One, 8, e64395. http://dx.doi.org/10.1371/journal.pone.0064395

[6] Erkan, A., Ekici, B., Ugurlu, M., Is, G., Seker, R., Demirtas, S. and Korkmaz, S. (2014) The Role of Bilirubin and Its Protective Function against Coronary Heart Disease. Herz, 39, 711-715. http://dx.doi.org/10.1007/s00059-013-3872-5

[7] Giraudi, P.J., Bellarosa, C., Coda-Zabetta, C.D., Peruzzo, P. and Tiribelli, C. (2011) Functional Induction of the Cystine-Glutamate Exchanger System Xc(-) Activity in SH-SY5Y Cells by Unconjugated Bilirubin. PloS One, 6, e29078. http://dx.doi.org/10.1371/journal.pone.0029078

[8] Huang, S.S., Huang, P.H., Wu, T.C., Chen, J.W. and Lin, S.J. (2012) Association of Serum Bilirubin with ContrastInduced Nephropathy and Future Cardiovascular Events in Patients Undergoing Coronary Intervention. PloS One, 7, e42594. http://dx.doi.org/10.1371/journal.pone.0042594

[9] Deetman, P.E., Bakker, S.J., Kwakernaak, A.J., Navis, G. and Dullaart, R.P. (2014) The Relationship of the AntiOxidant Bilirubin with Free Thyroxine Is Modified by Insulin Resistance in Euthyroid Subjects. PloS One, 9, e90886. http://dx.doi.org/10.1371/journal.pone.0090886

[10] Erdogan, D., Gullu, H., Yildirim, E., Tok, D., Kirbas, I., Ciftci, O., Baycan, S.T. and Muderrisoglu, H. (2006) Low Serum Bilirubin Levels Are Independently and Inversely Related to Impaired Flow-Mediated Vasodilation and Increased Carotid Intima-Media Thickness in Both Men and Women. Atherosclerosis, 184, 431-437. http://dx.doi.org/10.1016/j.atherosclerosis.2005.05.011

[11] Akboga, M.K., Canpolat, U., Sahinarslan, A., Alsancak, Y., Nurkoc, S., Aras, D., Aydogdu, S. and Abaci, A. (2015) Association of Serum Total Bilirubin Level with Severity of Coronary Atherosclerosis Is Linked to Systemic Inflammation. Atheroscleosis, 240, 110-114. http://dx.doi.org/10.1016/j.atherosclerosis.2015.02.051

[12] Ryu, S., Chang, Y., Zhang, Y., Woo, H.Y., Kwon, M.J., Park, H., Lee, K.B., Son, H.J., Cho, J. and Guallar, E. (2014) Higher Serum Direct Bilirubin Levels Were Associated with a Lower Risk of Incident Chronic Kidney Disease in Middle Aged Korean Men. PloS One, 9, e75178. http://dx.doi.org/10.1371/journal.pone.0075178

[13] Schwertner, H.A., Jackson, W.G. and Tolan, G. (1994) Association of Low Serum Concentration of Bilirubin with Increased Risk of Coronary Artery Disease. Clinical Chemistry, 40, 18-23.

[14] Ishizaka, N., Ishizaka, Y., Takahashi, E., Yamakado, M. and Hashimoto, H. (2001) High Serum Bilirubin Level Is Inversely Associated with the Presence of Carotid Plaque. Stroke: A Journal of Cerebral Circulation, 32, 580-583. http://dx.doi.org/10.1161/01.STR.32.2.580-b

[15] Perlstein, T.S., Pande, R.L., Beckman, J.A. and Creager, M.A. (2008) Serum Total Bilirubin Level and Prevalent Lower-Extremity Peripheral Arterial Disease: National Health and Nutrition Examination Survey (NHANES) 1999 to 2004. Arteriosclerosis, Thrombosis, and Vascular Biology, 28, 166-172. http://dx.doi.org/10.1161/ATVBAHA.107.153262

[16] Kimm, H., Yun, J.E., Jo, J. and Jee, S.H. (2009) Low Serum Bilirubin Level as an Independent Predictor of Stroke Incidence: A Prospective Study in Korean Men and Women. Stroke: A Journal of Cerebral Circulation, 40, 3422-3427. http://dx.doi.org/10.1161/STROKEAHA.109.560649

[17] Kuwano, T., Miura, S., Shirai, K., Ike, A., Mori, K., Shimizu, T., Zhang, B., Iwata, A., Nishikawa, H., Kawamura, A. and Saku, K. (2011) Serum Levels of Bilirubin as an Independent Predictor of Coronary In-Stent Restenosis: A New Look at an Old Molecule. Journal of Atherosclerosis and Thrombosis, 18, 574-583. http://dx.doi.org/10.5551/jat.6643

[18] Rothwell, P.M., Gutnikov, S.A. and Warlow, C.P. (2003) Reanalysis of the Final Results of the European Carotid Surgery Trial. Stroke: A Journal of Cerebral Circulation, 34, 514-523. http://dx.doi.org/10.1161/01.STR.0000054671.71777.C7 
[19] Li, S., Guo, Y.L., Xu, R.X., Zhang, Y., Zhu, C.G., Sun, J., Qing, P., Wu, N.Q., Jiang, L.X. and Li, J.J. (2014) Association of Plasma PCSK9 Levels with White Blood Cell Count and Its Subsets in Patients with Stable Coronary Artery Disease. Atherosclerosis, 234, 441-445. http://dx.doi.org/10.1016/j.atherosclerosis.2014.04.001

[20] Ishizaka, N., Ishizaka, Y., Toda, E., Nagai, R. and Yamakado, M. (2005) Association between Serum Uric Acid, Metabolic Syndrome, and Carotid Atherosclerosis in Japanese Individuals. Arteriosclerosis, Thrombosis, and Vascular Biology, 25, 1038-1044. http://dx.doi.org/10.1161/01.ATV.0000161274.87407.26

[21] Neuzil, J. and Stocker, R. (1994) Free and Albumin-Bound Bilirubin Are Efficient Co-Antioxidants for Alpha-Tocopherol, Inhibiting Plasma and Low Density Lipoprotein Lipid Peroxidation. The Journal of Biological Chemistry, 269, 16712-16719.

[22] Nakayama, M., Takahashi, K., Komaru, T., Fukuchi, M., Shioiri, H., Sato, K., Kitamuro, T., Shirato, K., Yamaguchi, T., Suematsu, M. and Shibahara, S. (2001) Increased Expression of Heme Oxygenase-1 and Bilirubin Accumulation in Foam Cells of Rabbit Atherosclerotic Lesions. Arteriosclerosis, Thrombosis, and Vascular Biology, 21, 1373-1377. http://dx.doi.org/10.1161/hq0801.093592

[23] Ollinger, R., Yamashita, K., Bilban, M., Erat, A., Kogler, P., Thomas, M., Csizmadia, E., Usheva, A., Margreiter, R. and Bach, F.H. (2007) Bilirubin and Biliverdin Treatment of Atherosclerotic Diseases. Cell Cycle, 6, 39-43. http://dx.doi.org/10.4161/cc.6.1.3700

[24] Vitek, L. and Schwertner, H.A. (2007) The Heme Catabolic Pathway and Its Protective Effects on Oxidative StressMediated Diseases. Advances in Clinical Chemistry, 43, 1-57. http://dx.doi.org/10.1016/S0065-2423(06)43001-8

[25] Ollinger, R., Bilban, M., Erat, A., Froio, A., McDaid, J., Tyagi, S., Csizmadia, E., Graca-Souza, A.V., Liloia, A., Soares, M.P., Otterbein, L.E., Usheva, A., Yamashita, K. and Bach, F.H. (2005) Bilirubin: A Natural Inhibitor of Vascular Smooth Muscle Cell Proliferation. Circulation, 112, 1030-1039. http://dx.doi.org/10.1161/CIRCULATIONAHA.104.528802

[26] Peyton, K.J., Shebib, A.R., Azam, M.A., Liu, X.M., Tulis, D.A. and Durante, W. (2012) Bilirubin Inhibits Neointima Formation and Vascular Smooth Muscle Cell Proliferation and Migration. Frontiers in Pharmacology, 3, 48. http://dx.doi.org/10.3389/fphar.2012.00048

[27] Yang, X.F., Chen, Y.Z., Su, J.L., Wang, F.Y. and Wang, L.X. (2009) Relationship between Serum Bilirubin and Carotid Atherosclerosis in Hypertensive Patients. Internal Medicine, 48, 1595-1599. http://dx.doi.org/10.2169/internalmedicine.48.2286

[28] Stocker, R., Yamamoto, Y., McDonagh, A.F., Glazer, A.N. and Ames, B.N. (1987) Bilirubin Is an Antioxidant of Possible Physiological Importance. Science, 235, 1043-1046. http://dx.doi.org/10.1126/science.3029864

[29] Frei, B., Stocker, R. and Ames, B.N. (1988) Antioxidant Defenses and Lipid Peroxidation in Human Blood Plasma. Proceedings of the National Academy of Sciences of the United States of America, 85, 9748-9752. http://dx.doi.org/10.1073/pnas.85.24.9748

[30] Yamaguchi, T., Terakado, M., Horio, F., Aoki, K., Tanaka, M. and Nakajima, H. (1996) Role of Bilirubin as an Antioxidant in an Ischemia-Reperfusion of Rat Liver and Induction of Heme Oxygenase. Biochemical and Biophysical Research Communications, 223, 129-135. http://dx.doi.org/10.1006/bbrc.1996.0857

[31] Trappolini, M., Stoppo, M., Meggiorini, M., Loguercio, V., Fabiani, O., Iannotta, M., Negro, A., Sebastianelli, A., Proietta, M., Del Porto, F. and Aliberti, G. (2009) The Plasma Fibrinogen as a Marker of Subclinical Carotid Atherosclerosis. Recenti Progressi in Medicina, 100, 352-355.

[32] Redgrave, J.N., Lovett, J.K. and Rothwell, P.M. (2010) Histological Features of Symptomatic Carotid Plaques in Relation to Age and Smoking: The Oxford Plaque Study. Stroke: A Journal of Cerebral Circulation, 41, 2288-2294. http://dx.doi.org/10.1161/STROKEAHA.110.587006 